\title{
Anticholinergic burden in older women: not seeing the wood for the trees?
}

\author{
A high \\ anticholinergic \\ medicines \\ burden in this \\ group was \\ driven by the \\ use of multiple \\ medicines \\ with lower \\ anticholinergic \\ potency rather \\ than the use of \\ medicines with \\ higher potency
}

Lynne Parkinson

$\mathrm{BSC}$ (Hons), PhD

Parker J Magin

PhD, FRACGP

Allison Thomson
BMed $^{3}$

Julie E Byles BMed, $\mathrm{PhD}^{2}$

Gillian E Caughey

$\mathrm{PhD}$

Christophe Etherton-Bee MBBS, PhD, FRACP5,

Danijela Gnjidic $\mathrm{BSc}$ (Hons), PhD, MPH

Sarah N Hilmer MBBS(Hons), FRACP, PhD ${ }^{7,8}$

Thomas King Tong Lo MClinEpi, BScH, BA

Colin McCowan BSc, MSc, $P h D^{9}$

Rachael Moorin

$\mathrm{PhD}^{10}$

C Dimity Pond MBBS, FRACGP, PhD²
O lder people are particularly vulnerable to adverse medicines-related events. Reasons for this include the physiological changes of ageing, the chronic and comorbid conditions they often have, the types of medicines they are commonly prescribed, and the frequency with which they use multiple medicines. ${ }^{1,2}$ Adverse effects related to medicine use are a significant health problem in this growing population group. ${ }^{3}$ Many medicines used by older people have anticholinergic effects (effects through one of the body's principal neurotransmitter systems). ${ }^{4}$ The anticholinergic effect of an individual medicine may be small, but the anticholinergic effects of multiple medicines may be additive, constituting "anticholinergic burden". ${ }^{1,4,5}$

The degree of anticholinergic effect varies greatly between drugs and drug classes. ${ }^{6}$ Drug classes with anticholinergic effects that are commonly used by older people include gastrointestinal antispasmodics, medicines used for urge incontinence, antipsychotics, and tricyclic antidepressants. ${ }^{4}$ The anticholinergic effect may be intrinsic to the therapeutic effect of the medicine or an unintended side effect. ${ }^{4}$

Relatively minor anticholinergic adverse effects that are readily apparent include dry mouth, constipation and blurred vision. ${ }^{4}$ More serious effects, such as confusion and impaired cognition, have also been consistently associated with anticholinergic medicines. ${ }^{7-10}$ Anticholinergic burden can also affect functional status in older people, generally related to instrumental activities of daily living (skills necessary for individuals to live independently); ${ }^{1,7}$ balance, gait and mobility; ${ }^{5}$ and is associated with falls and frailty.,11

An Australian study found that $21 \%$ of community-dwelling men aged 70 years or older were taking medicines with definite anticholinergic effects. ${ }^{1}$

\section{Abstract}

Objectives: To identify medicines contributing to and describe predictors of anticholinergic burden among community-dwelling older Australian women.

Design, setting and participants: Retrospective longitudinal analysis of data from the Australian Longitudinal Study on Women's Health linked to Pharmaceutical Benefits Scheme medicines data from 1 January 2008 to 30 December 2010; for 3694 women born in 1921-1926.

Main outcome measures: Anticholinergic burden calculated from Anticholinergic Drug Scale (ADS) scores derived from ADS levels (0 to 3 ) for all medicines used by each woman, summed over each 6-month period (semester), medicines commonly used by women with high semester ADS scores (defined as 75th percentile of scores).

Results: 1126 women (59.9\%) used at least one medicine with anticholinergic properties. The median ADS score was 4 or 5 across all semesters. Most anticholinergic medicines used by women who had a high anticholinergic burden (ADS score, $\geqslant 9$ ) had a low anticholinergic potency (ADS level 1). Increasing age, cardiovascular disease, and number of other medicines used were predictive of a higher anticholinergic burden.

Conclusions: A high anticholinergic medicines burden in this group was driven by the use of multiple medicines with lower anticholinergic potency rather than the use of medicines with higher potency. This is a novel and important finding for clinical practice as doctors would readily identify the risk of a high anticholinergic burden for patients using high potency medicines, but may be less likely to identify this risk for users of multiple medicines with low anticholinergic potency.

Although women outnumber men in the older population, ${ }^{3}$ research into anticholinergic medicine use has focused predominantly on mixed sex and male samples. ${ }^{1,8,11}$ However, United States studies have shown that $15 \%$ of women aged 75 years and older ${ }^{12}$ and $11 \%$ of women aged 50-79 years $^{13}$ used anticholinergic medicines.

Women may have a particular propensity to be prescribed highly anticholinergic medicines for conditions such as urinary incontinence and chronic neuropathic pain, and they use an increasing number of medicines as they age. The cumulative anticholinergic effects of these medicines can increase the risk of serious functional impairment, negatively affecting independence in older age. In this study we aimed, for the period 2008 to 2010, to:

- describe the anticholinergic medicine burden among a community-based sample of older women from the Australian
Longitudinal Study on Women's Health (ALSWH);

- identify medicines and combinations of medicines that make the greatest contribution to anticholinergic burden; and

- describe the predictors of high anticholinergic medicine burden.

\section{Methods}

In this retrospective observational longitudinal study, we analysed survey data from the ALSWH, linked to Pharmaceutical Benefits Scheme (PBS) data. Ethics approval for data collection, linkage and analyses was obtained through the University of Queensland, University of Newcastle and Australian Department of Health and Ageing.

\section{Sample}

The sample included women from the ALSWH "older" cohort (born in 1921-1926), who had completed Survey 5 (2008), consented to PBS 
1 Central Queensland University,

North Rockhampton, QLD.

2 University of Newcastle. Newcastle, NSW.

3 General Practice Training Valley to Coast.

Newcastle, NSW.

4 Sansom Institute for Health Research,

University of South

Australia,

Adelaide, SA

5 University of

Western Australia,

Perth, WA.

6 Royal Perth Hospital, Perth, WA

7 The University of Sydney,

Sydney, NSW.

8 Royal North Shore Hospital, Sydney, NSW.

9 University of Glasgow, Glasgow,

United Kingdom.

10 Curtin University, Perth, WA

l.parkinson@cqu.edu.au

doi: 10.5694/mjal4.00336

linkage, had concessional status for the PBS and had made at least one medicine claim from 1 January 2008 to 30 December 2010.

\section{Data sources}

ALSWH survey data: The ALSWH is a national study that began in 1996 with a random sample of more than 40000 women in three birth cohorts. Here, we focus on those in the older cohort, born in 1921-1926, who completed Survey 1 in 1996. Since 1998, follow-up surveys have been completed every 3 years. The older cohort were aged 70-75 years at Survey 1 (12432 women) and 82-87 years at Survey 5 in 2008 (5560 women). Deaths are ascertained using the National Death Index.

Compared with the general population, women in the cohort have had a small relative survival advantage, mainly due to baseline demographic and health behaviour differences. Compared with national data, underrepresentation of women born in nonEnglish-speaking countries and those who were underweight has increased slightly over the course of the study. ${ }^{14}$ Such small biases are unlikely to affect measures of association.

The surveys covered a range of health, social, psychological and demographic variables. Detailed methods and surveys are available from the ALSWH website (http:// www.alswh.org.au).

Medicines data: These were records of subsidised prescriptions under the PBS and Repatriation PBS (RPBS) provided by Medicare Australia, for the calendar years 2008 through 2010. These records provide reliable data about dates of services and medicine types. The ALSWH study uses deterministic linkage between survey and individual PBS data, using personal identifier numbers held by Medicare.

PBS data include only PBS-listed prescription medicines that attract a government subsidy, so they do not include medicines provided in hospital or purchased over-the-counter (OTC). Although OTC medicines with anticholinergic activity are not captured by the PBS, we did not expect a significant impact on our results, as at
ALSWH Survey 4 (2005), less than 5\% of women in the older cohort reported OTC medicine use. ${ }^{15}$ Medicines data for those with concessional status are captured consistently by PBS, as all PBS medicines cost more than the concessional status threshold and will always attract a government subsidy.

\section{Statistical analyses}

PBS data were coded to conform to Anatomical Therapeutic Chemical Codes. ${ }^{16}$

Anticholinergic medicines were identified and their potency rated using the Anticholinergic Drug Scale (ADS) ${ }^{6}$ as follows.

Level 1: potentially anticholinergic as evidenced by receptor binding studies (for example, frusemide, digoxin, or captopril).

Level 2: anticholinergic adverse events sometimes noted, usually at excessive doses (for example, carbamazepine, cyproheptadine, or disopyramide).

Level 3: markedly anticholinergic (for example, amitriptyline, brompheniramine, or oxybutynin). ${ }^{6}$

The ADS provides a measure of anticholinergic burden assessed from serum anticholinergic activity for individual medicines; ${ }^{6}$ and has recently been shown to predict adverse medicines-related events. ${ }^{17}$

The characteristics of women who used at least one anticholinergic medicine at any time from 2008 through 2010 were compared with those who did not use any, using $\chi^{2}$ tests for categorical variables and two-sided $t$ tests for continuous variables.

The ADS potency ratings (level 1, 2 or 3) of all anticholinergic medicines claims for each woman were summed to give individual 6-month anticholinergic burden (ADS) scores (semesters were January to June and July to December each year), for 2008 to 2010. We did not consider medicine doses in calculating ADS scores, as it has been shown that including the dose does not improve ADS correlation with serum anticholinergic activity. ${ }^{6}$
ADS scores were tabulated and graphed. High ADS scores were those in the 75th percentile of all scores, and above. Anticholinergic medicines associated with high 6-month ADS scores were identified.

The predictors of high 6-month ADS scores for women using anticholinergic medicines were analysed using stepwise backwards generalised estimating equations (GEE), with the significance level set at $P<0.05$. Women who made no claims for anticholinergic medicines were excluded as they are likely to be a different population to those who receive at least one anticholinergic medicine, and including them would erroneously intensify differences between groups. Sociodemographic covariates considered in the model were: age, area of residence (rural/urban), education level (secondary or above/ below secondary), living arrangements (alone/with others), marital status (partnered/not). Lifestyle covariates were: smoking status (current/not), alcohol use (yes/no), body mass index (mean).$^{18}$ Health covariates included: conditions (yes/no for mental health problems [depression, anxiety, or nervous disorder], cardiovascular disease [heart attack, other heart problems, or stroke], diabetes, arthritis, asthma, cancer [excluding skin cancer], and osteoporosis), total number of other (not anticholinergic) medicines, quality of life (measured by the 36-item short form health survey [SF-36]). ${ }^{19}$

All statistical analyses were performed in Stata/IC, version 11 (StataCorp). Data file construction was performed using SAS, version 9.4 (SAS Institute).

\section{Results}

\section{Sample characteristics}

There were 5560 women born in 19211926 who returned ALSWH Survey 5; 3694 of these $(66.4 \%)$ consented to PBS linkage (for 2008, 2009, and 2010). Of those $3694,1883(51.0 \%)$ had at least one PBS claim from 2008 to 2010, and had concessional PBS status. Of these women, $1126(59.8 \%)$ had at least one 
1 Claims for anticholinergic medicines (AM) by Australian women aged 82-89 years, annually and 6-monthly, 2008 through 2010

\begin{tabular}{|c|c|c|c|c|c|c|c|c|c|c|}
\hline \multirow[b]{2}{*}{ Year } & \multirow{2}{*}{$\begin{array}{l}\text { Women with any } \\
\text { medicine claim }\end{array}$} & \multirow{2}{*}{$\begin{array}{l}\text { Women with } \\
\text { AM claim }\end{array}$} & \multicolumn{3}{|c|}{ AM claims by ADS level* } & \multicolumn{5}{|c|}{ Number of AM claims } \\
\hline & & & Level 1 & Level 2 & Level 3 & Min & Q1 & Median & Q3 & Max \\
\hline \multicolumn{11}{|l|}{2008} \\
\hline Annual & 1844 & $783(42.5 \%)$ & 687 & 16 & 174 & 1 & 2 & 6 & 12 & 56 \\
\hline Jan-Jun & 1822 & $633(34.7 \%)$ & 555 & 13 & 130 & 1 & 2 & 4 & 7 & 33 \\
\hline Jul-Dec & 1808 & $681(37.7 \%)$ & 593 & 13 & 147 & 1 & 2 & 4 & 7 & 27 \\
\hline \multicolumn{11}{|l|}{2009} \\
\hline Annual & 1812 & 796 (43.9\%) & 694 & 16 & 187 & 1 & 2 & 6 & 12 & 44 \\
\hline Jan-Jun & 1786 & $654(36.6 \%)$ & 566 & 11 & 142 & 1 & 2 & 4 & 7 & 37 \\
\hline Jul-Dec & 1768 & $683(38.6 \%)$ & 592 & 13 & 149 & 1 & 2 & 4 & 7 & 27 \\
\hline \multicolumn{11}{|l|}{2010} \\
\hline Annual & 1755 & $823(46.9 \%)$ & 733 & 21 & 169 & 1 & 2 & 6 & 13 & 50 \\
\hline Jan-Jun & 1734 & 677 (39.0\%) & 594 & 17 & 135 & 1 & 2 & 4 & 7 & 24 \\
\hline Jul-Dec & 1702 & 706 (41.5\%) & 628 & 13 & 137 & 1 & 2 & 4 & 7 & 26 \\
\hline
\end{tabular}

anticholinergic medicine claim from 2008 to 2010.

Appendix 1 shows that, compared with women who had no anticholinergic medicines claims, the group with anticholinergic medicines claims had: a lower proportion of women with a secondary education or higher $(P<0.05)$; a higher mean BMI $(P<0.05)$; a higher proportion with cancer $(P<0.05)$, a mental health problem $(P<0.001)$, asthma $(P<0.001)$, cardiovascular disease $(P<0.001)$, and arthritis $(P<0.001)$; higher multimorbidity $(P<0.001)$. They also performed less well on all SF-36 scales $(P<0.001)$

\section{Use of anticholinergic medicines}

Fifty different anticholinergic medicines were used by this group (21 at ADS level 3, four at ADS level 2 and 25 at ADS level 1). The proportion of women who used anticholinergic medicines increased over the 3 years, from $783(42.5 \%)$ in 2008 to 823 (46.9\%) in 2010 (Box 1). Just over a third of women using anticholinergic medicines $(34.3 \%)$ used these in all six semesters, $25.7 \%$ used them in only one, $14.7 \%$ used them in two, $10.8 \%$ in three, $7.9 \%$ in four, and $6.8 \%$ in five semesters. The median number of claims for anticholinergic medicines per woman was four (interquartile range [IQR], 2-7) in all semesters. Most anticholinergic medicines used were at ADS level 1.

2 Anticholinergic drug scale scores for 1126 Australian women aged 82-89 years who used anticholinergic medicines (AM), by 6-month semester, 2008 through 2010

\begin{tabular}{|c|c|c|c|c|c|c|c|}
\hline \multirow[b]{2}{*}{ Year } & \multirow[b]{2}{*}{ Semester } & \multirow{2}{*}{$\begin{array}{l}\text { Women with } \\
\text { AM claim }\end{array}$} & \multicolumn{5}{|c|}{ Anticholinergic drug scale scores } \\
\hline & & & Minimum & Q1 & Median & Q3 & Maximum \\
\hline \multirow[t]{2}{*}{2008} & Jan-Jun & 633 & 1 & 2 & 5 & 9 & 33 \\
\hline & Jul-Dec & 681 & 1 & 2 & 5 & 9 & 30 \\
\hline \multirow[t]{2}{*}{2009} & Jan-Jun & 654 & 1 & 2 & 4 & 8 & 37 \\
\hline & Jul-Dec & 683 & 1 & 2 & 5 & 8 & 39 \\
\hline \multirow[t]{2}{*}{2010} & Jan-Jun & 677 & 1 & 2 & 5 & 8 & 39 \\
\hline & Jul-Dec & 706 & 1 & 2 & 5 & 9 & 33 \\
\hline
\end{tabular}

\section{Anticholinergic burden (ADS score)}

ADS scores were relatively stable across all semesters over the 3 years, as shown in Box 2. Median ADS scores were 4 or 5 in all semesters. A high ADS score for a semester was defined as $\geqslant 9$ (75th percentile of scores).

\section{Main contributors to anticholinergic burden}

Most anticholinergic medicines used by women with high ADS scores were at ADS level 1. Given the diversity of ADS medicines used, we identified no common combinations of medicines contributing to high ADS scores. The 10 most commonly used anticholinergic medicines in women with high ADS scores were very similar across the six semesters: amitriptyline (level 3), digoxin (level 1), doxepin (level 3), frusemide (level 1), isosorbide (level 1), nifedipine (level 1), oxycodone (level 1), prednisolone (level 1), and warfarin (level 1) were present in each semester, with fentanyl (level 1) in four and dothiepin (level 3) in two semesters (Appendix 2).

\section{Characteristics of older women with a high anticholinergic burden}

The final parsimonious GEE model showed that increasing age, selfreport of cardiovascular disease (heart attack, other heart problems, and stroke) and number of other (not anticholinergic) medicines were predictive of a higher anticholinergic burden for women using anticholinergic medicines (Box 3). 
3 Multivariate generalised estimating equation regression of explanatory variables (final model) for anticholinergic medicine users with a high anticholinergic burden (ADS score $\geqslant 9$ ) compared with those with lower anticholinergic burden (ADS score <9)

\begin{tabular}{lccc} 
Variable & Odds ratio $(95 \% \mathrm{CI})$ & SE & $P$ \\
\hline Age & $1.05(1.01-1.08)$ & 0.02 & $0.006 *$ \\
Education ( secondary) & $0.85(0.72-1.00)$ & 0.07 & 0.056 \\
Alcohol use & $0.86(0.73-1.02)$ & 0.07 & 0.089 \\
Cardiovascular disease & $1.25(1.05-1.47)$ & 0.11 & $0.010 *$ \\
SF-36 pain index & $1.00(1.00-1.01)$ & 0.00 & 0.047 \\
SF-36 general health perception & $0.99(0.99-1.00)$ & 0.00 & $0.005 *$ \\
Number of non-anticholinergic medicines & $1.05(1.04-1.06)$ & 0.01 & $0.000 *$ \\
\hline
\end{tabular}

ADS = anticholinergic drug scale. SF-36 = 36-item short form health survey.

* Significant difference $(P<0.05)$.
Our study has some potential limitations. While the PBS provides comprehensive data, as outlined in the methods, it does not include all medicines (eg, OTC medicines). Although we expect this would not have significantly affected our findings, this limitation may mean that our calculation of burden is conservative. Also, as medicines costing less than the patient "copayment" will not be captured in the PBS, ${ }^{24}$ we restricted our analyses to women with "concessional" status, as is common practice. ${ }^{2}$ As the vast majority of older women have concessional status, the effect on the generalisability of our findings to this group should be limited.

This study showed that a high proportion of older women have a substantial anticholinergic burden, and that this high burden is driven by the use of multiple medicines with lower anticholinergic potency rather than use of medicines with higher anticholinergic potency. Our study is one of the few in Australia and internationally that evaluate anticholinergic burden in older women. It is also unique in that it examines anticholinergic burden longitudinally, using continuous and reliable data on medicines from the PBS.

While there are many methods for measuring anticholinergic burden, ${ }^{4,20}$ we used the ADS, which provides a measure of anticholinergic burden that correlates well with serum anticholinergic activity measures. ${ }^{6}$ A US clinic-based study of women aged 75 years and older, using a similar measure of anticholinergic burden, found that mean burden increased significantly in the 10 years of their study; however, only $15 \%$ of participants were using anticholinergic medicines within the last month at follow-up. ${ }^{12}$ Another US study using data from the Women's Health Initiative found that $11 \%$ of women aged 50-79 years were using an anticholinergic medicine at baseline. ${ }^{13}$ Although we found that median anticholinergic burden did not increase over time, 35\% of our community sample were using anticholinergic medicines during the 6-month baseline semester, and this proportion increased significantly over time. Over a third of women using anticholinergic medicines used these in all semesters, suggesting continuous use.

Total avoidance of medicines with anticholinergic properties for older people is neither practicable nor necessarily desirable. ${ }^{21}$ Multiple medicines use is not only common among older adults, but is important for ameliorating symptoms, improving quality of life, and sometimes for curing disease. ${ }^{21}$ Individual prescribing decisions about medicines with anticholinergic activity, as with all medicines, will always involve assessing the potential benefits and harms ${ }^{22}$ and, in past research, we showed that anticholinergic burden is an area where assessing the risk is problematic. ${ }^{23}$ In interviews with Australian general practitioners we found that they have limited understanding of the concept of anticholinergic burden and the range of medicines that contribute to it, despite otherwise having a sophisticated understanding of potential medicine adverse events and managing this risk.

The predictors of a high anticholinergic burden were generally not unexpected for the group we studied. One of our aims in this analysis was to identify characteristics of older people that might alert doctors to a particular risk of higher anticholinergic medicine burden. While unremarkable predictors may not be very informative in clinical practice, our study does emphasise that identifying a higher anticholinergic medicine burden is complex, and there appear to be no simple flags to help doctors identify the risk.

\section{Conclusions}

It is a novel and important finding for clinical practice that high anticholinergic medicines burden in this group was driven by the use of multiple medicines with lower anticholinergic potency rather than by those with higher anticholinergic potency. While we might expect that doctors would readily identify anticholinergic burden as a risk in patients using medicines with high anticholinergic potency, they may be less likely to perceive a risk for patients using multiple medicines with lower anticholinergic potency. Developing a means of calculating the anticholinergic burden of drug regimens (and of the contributions of individual drugs) and incorporating this into GPs' prescribing software would be appropriate. Our findings provide important evidence to underpin prescribing practice and policy aimed at reducing disability and adverse medicine events among older women, and may apply to all older people.

Acknowledgements: This report is based on research conducted as part of the Australian Longitudinal Study on Women's Health, the University of Newcastle and the University of Queensland. We are grateful to the Australian Government Department of Health and Ageing for funding and to the women who provided the survey data. We gratefully acknowledge funding from the RACGP Foundation for analyses for this project. We acknowledge Medicare Australia for the Pharmaceutical Benefits Scheme data. This report was supported by the Australian Government's Collaborative Research Networks (CRN) Program. Danijela Gnjidic is supported by a National Health and Medical Research Council Early Career Fellowship.

Competing interests: No relevant disclosures.

Received 10 Mar 2014, accepted 8 Aug 2014. - 
1 Gnjidic D, Cumming RG, Le Couteur DG, et al. Drug Burden Index and physical function in older Australian men. $\mathrm{Br} J$ Clin Pharmacol 2009; 68: 97-105.

2 Roughead EE, Kalisch LM, Barratt JD, Gilbert AL. Prevalence of potentially hazardous drug interactions amongst Australian veterans. Br J Clin Pharmacol 2010; 70: 252-257.

3 Australian Institute of Health and Welfare. Older Australia at a glance. 4th ed. Canberra: AlHW, 2007. http:// www.aihw.gov.au/publicationdetail/?id=6442468045 (accessed Aug 2014).

4 Bostock CV, Soiza RL, Mangoni AA. Association between prescribing of antimuscarinic drugs and antimuscarinic adverse effects in older people. Expert Rev Clin Pharmacol 2010; 3: 441-452.

5 Cao YJ, Mager DE, Simonsick EM, et al. Physical and cognitive performance and burden of anticholinergics, sedatives, and ACE inhibitors in older women. Clin Pharmacol Ther 2008; 83: 422-429.

6 Carnahan RM, Lund BC, Perry PJ, et al. The Anticholinergic Drug Scale as a measure of drug-related anticholinergic burden: associations with serum anticholinergic activity. $J$ Clin Pharmacol 2006; 46: 1481-1486.

7 Hilmer SN, Mager DE, Simonsick EM, et al. A drug burden index to define the functional burden of medications in older people. Arch Intern Med 2007; 167: 781-787.

8 Fox C, Richardson K, Maidment ID, et al. Anticholinergic medication use and cognitive impairment in the older population: the medical research council cognitive function and ageing study. J Am Geriatr Soc 2011; 59: 1477-1483.

9 Gnjidic D, Le Couteur DG, Naganathan $\checkmark$, et al. Effects of drug burden index on cognitive function in older men. J Clin Psychopharmacol 2012; 32: 273-277.

10 Kashyap M, Belleville S, Mulsant BH, et al. Methodological challenges in determining longitudinal associations between anticholinergic drug use and incident cognitive decline. J Am Geriatr Soc 2014; 62: 336-341.

1 Wilson NM, Hilmer SN, March LM, et al. Associations between drug burden index and falls in older people in residential aged care. J Am Geriatr Soc 2011; 59: 875-880.

12 Koyama A, Steinman M, Ensrud K, et al. Ten-year trajectory of potentially inappropriate medications in very old women: importance of cognitive status. J Am Geriatr Soc 2013; 61: 258-263.

13 Wirtz HS, Gray S, Pettinger MC, et al. Anticholinergic medication use, falls and fracture in postmenopausal women: Results from the women's health initiative. J Am Geriatr Soc 2012; 60 Suppl 4: SII-SI2.

14 Byles JE, Dobson A. The value of time in longitudinal studies of ageing. Lessons from the Australian Longitudinal Study on Women's Health. Australas J Ageing 2011; 30 Suppl 2: 6-12.

15 Byles J, Loxton D, Berecki J, et al. Use and costs of medications and other health care resources: findings from the Australian Longitudinal Study on Women's Health. Report prepared for the Australian Government Department of Health and Ageing. Newcastle: ALSWH, 2008. http://www. alswh.org.au/images/content/pdf/ major_reports/2008_major_report_c_ r144.pdf (accessed Aug 2014).

16 World Health Organization Collaborating Centre for Drug Statistics Methodology. The anatomical therapeutic chemical classifications system with defined daily doses (ATC/ DDD) Index 2013. Geneva: WHO, 2013. http://www.who.int/classifications/ atcddd/en (accessed Feb 2014).

17 Lampela P, Lavikainen, P, GarciaHorsman JA, et al. Anticholinergic drug use, serum anticholinergic activity, and adverse drug events among older people: a population-based study. Drugs Aging 2013; 30: 321-330.

18 World Health Organization Global Health Observatory. Mean body mass index (BMI). Geneva: WHO, 2013. http://www.who.int/gho/ncd/ risk_factors/bmi_text/en/index.html (accessed Feb 2014).

19 Ware JE Jr, Sherbourne CD. The MOS 36-item short form health survey (SF36): I. Conceptual framework and item selection. Med Care 1992; 30: 473-483.

20 Hilmer SN, Gnjidic D, Abernethy DR. Pharmacoepidemiology in the postmarketing assessment of the safety and efficacy of drugs in older adults. J Gerontol A Biol Sci Med Sci 2012; 67: 181-188.

21 Steinman MA, Hanlon JT. Managing medications in clinically complex elders: "There's got to be a happy medium". JAMA 2010; 304: 1592-1601.

22 Egger SS, Bachmann A, Hubmann $\mathrm{N}$, et al. Prevalence of potentially inappropriate medication use in elderly patients: comparison between general medical and geriatric wards. Drugs Aging 2006; 23: 823-837.

23 Magin P, Goode S, Pond D. GPs, medications and older people: a qualitative study of general practitioners' approaches to potentially inappropriate medications in older people. Australas J Ageing 2014; 23 Apr [Epub ahead of print]. doi: 10.1111/ajag.12150.

24 Smith A, Tett SE. How do different age groups use benzodiazepines and antidepressants? Analysis of an Australian administrative database, 2003-6. Drugs Aging 2009; 26: 113-1 E 